\title{
Diophantine properties of real numbers generated by finite automata
}

\author{
Boris Adamczewski and Julien Cassaigne
}

\begin{abstract}
We study some Diophantine properties of automatic real numbers and we present a method to derive irrationality measures for such numbers. As a consequence, we prove that the $b$-adic expansion of a Liouville number cannot be generated by a finite automaton, a conjecture due to Shallit.
\end{abstract}

\section{Introduction}

The seminal work of Turing [Tur37] gives rise to a rough classification of real numbers. On one side we find computable real numbers, that is, real numbers whose binary (or more generally $b$-adic) expansion can be produced by a Turing machine, while on the other side lie uncomputable real numbers which, in some sense, 'escape to computers'. Although most real numbers belong to the second class (the first being countable), classical mathematical constants are usually computable. By classical constants, we mean real numbers such as $\sqrt{2}$ (or more generally algebraic real numbers), $\pi$ and $\zeta(3)$. Note that the notion of period as considered by Kontsevitch and Zagier [KZ01] could offer an interesting framework for (most of) these mathematical constants. Following the pioneering ideas of Turing, Hartmanis and Stearns [HS65] proposed to emphasize the quantitative aspect of the notion of computability, and to take into account the number $T(n)$ of operations needed by a (multitape) Turing machine to produce the first $n$ digits of the expansion. In this regard, a real number is considered all the more simple as its $b$-adic expansion can be produced very fast by a Turing machine. A general problem is then to determine where our mathematical constants take place in such a classification. It is a source of challenging open questions such as the HartmanisStearns problem which asks whether there exists an irrational algebraic number computable in real-time, that is, with $T(n)=O(n)$. In the same spirit, we mention [BT98] where similar questions are addressed for formal power series with coefficients in a finite field.

The present paper is partly motivated by the related question of how such a classification of real numbers based on Turing machines and theoretical computer science fits into that based on Diophantine approximation which was developed by Mahler [Mah31, Mah32a] during the 1930s. More modestly, we will focus on a special class of Turing machines of a particular interest: finite automata. They are one of the most basic models of computation and thus take place at the bottom of the hierarchy of Turing machines. In particular, such machines produce sequences in real-time. Roughly speaking, an infinite sequence $\mathbf{a}=\left(a_{n}\right)_{n \geqslant 0}$ is generated by a $k$-automaton if $a_{n}$ is a finitestate function of the base- $k$ representation of $n$. Consequently, automatic sequences share deep links with number theory as explained in detail in the book of Allouche and Shallit [AS03].

A real number is generated by a finite automaton (or, in short, automatic), if there exists a positive integer $b \geqslant 2$ such that its $b$-adic expansion can be generated by a finite automaton. Particular

Received 23 January 2006, accepted in final form 4 April 2006.

2000 Mathematics Subject Classification 11B85 (primary), 11J82 (secondary).

Keywords: automatic sequences, Liouville's numbers, measures of irrationality, Mahler's classification.

This journal is (c) Foundation Compositio Mathematica 2006. 


\section{B. Adamczewsiki and J. Cassaigne}

attention was paid to these numbers since the following statement related to the Hartmanis-Stearns problem was suggested by Cobham [Cob68] in 1968: no irrational algebraic number can be generated by a finite automaton. Previously referred to as the Loxton-van der Poorten conjecture because of some attempts by these authors (see, for instance, [LV82, LV88]), this result was recently proved in [AB, ABL04]. However, it is still unknown whether constants such as $\pi, \zeta(3)$ or $e$ are automatic.

Our main purpose is to introduce a method to derive Diophantine properties for all automatic real numbers. This uses some of the ideas in [AB, ABL04] together with classical techniques from Diophantine approximation and a careful combinatorial study of automatic sequences. In particular, we will prove that no Liouville number can be generated by a finite automaton, a result conjectured by Shallit [Sha99]. Actually, our approach is much more precise and it will provide an explicit general upper bound for the irrationality measure of any automatic real number.

This article is organized as follows. We first present our main results, including a proof of Shallit's conjecture, in $\S 2$. In $\S 3$, we state a partial result towards a more general conjecture on automatic real numbers. This conjecture suggested by Becker involves Mahler's classification of real numbers and we will thus briefly recall some facts about it. A background on finite automata, words and morphisms can be found in $\S 4$. Section 5 is devoted to the proofs of our main results. As an application of our method, we derive an irrationality measure for the Thue-Morse-Mahler numbers in $\S 6$. Some comments and generalizations are gathered in $\S 7$. Finally, outlines of the proofs of these latter results end this paper.

\section{Main results}

Diophantine approximation is essentially devoted to the estimate of the approximation of a given real number by rationals $p / q$, as a function of $q$. A useful notion to tackle this question is the irrationality measure of a real number $\xi$, that we will denote by $\mu(\xi)$. It is defined as the supremum of the positive real numbers $\tau$ for which the inequality

$$
\left|\xi-\frac{p}{q}\right|<\frac{1}{q^{\tau}}
$$

has infinitely many solutions $(p, q) \in \mathbb{Z}^{2}$ with $q>1$ and relatively prime integers $p$ and $q$. Thus, $\mu(\xi)$ measures the quality of the best rational approximations to $\xi$. Note that it is in general a challenging problem to obtain an irrationality measure, that is, to bound from above the irrationality measure of a given real number.

Let us recall some well-known facts about this notion. First, the theory of continued fractions ensures that $\mu(\xi) \geqslant 2$, for any irrational number $\xi$; while $\mu(\xi)=1$ for a rational number $\xi$. On the other hand, algebraic irrational numbers all have an irrationality measure equal to two, as follows from Roth's theorem [Rot55]. Note that this is also the case for almost all real numbers (with respect to the Lebesgue measure), a result due to Khintchine [Khi24] (see also [Khi64]).

At the opposite side, we find the Liouville numbers, introduced by Liouville in his famous 1844 paper [Lio44]. Such numbers can be very well approximated by rationals. In more concrete terms, an irrational real number $\xi$ is called a Liouville number if it has an infinite irrationality measure, that is, if for any positive $\tau$ the inequality

$$
\left|\xi-\frac{p}{q}\right|<\frac{1}{q^{\tau}}
$$

has at least one solution $(p, q) \in \mathbb{Z}^{2}$ with $q>1$.

Our main result gives a positive answer to a conjecture of Shallit [Sha99].

Theorem 2.1. A Liouville number cannot be generated by a finite automaton. 


\section{Diophantine PROPERTIES OF AUTOMATIC REAL NUMBERS}

Actually, we will prove a quantitative version of Theorem 2.1. Indeed, our method provides the following explicit general upper bound for the irrationality measure of any number generated by a finite automaton. This bound only depends on three parameters which naturally appear in the study of automatic sequences and whose definitions are postponed to $\S 4$.

Theorem 2.2. Let $k$ and $b$ be two integers at least equal to two and let $\mathbf{a}=\left(a_{n}\right)_{n \geqslant 0}$ be an infinite sequence generated by a $k$-automaton and with values in $\{0,1, \ldots, b-1\}$. Let $m$ be the cardinality of the $k$-kernel of the sequence a and let $d$ be the cardinality of the internal alphabet associated to a. Then, the irrationality measure $\mu(\xi)$ of the real number

$$
\xi:=\sum_{n=0}^{+\infty} \frac{a_{n}}{b^{n}}
$$

satisfies

$$
\mu(\xi) \leqslant d k\left(k^{m}+1\right)
$$

\section{The classification of Mahler}

In 1932, Mahler [Mah31, Mah32a] introduced the first relevant classification of real numbers with respect to Diophantine approximation or more precisely with respect to their quality of approximation by algebraic numbers. According to Mahler's classification, real numbers are split into four classes, namely $A$-numbers, $S$-numbers, $T$-numbers, and $U$-numbers. We recall now how these four classes can be defined as first considered by Mahler. The reader is referred to [Bug04], for example, for a complete treatment of this topic.

Let $n$ be a positive integer and $\xi$ be a real number. Then, we define $w_{n}(\xi)$ as the supremum of the real numbers $\omega$ for which there exist infinitely many integer polynomials $P(X)$ of degree at most $n$ and such that

$$
0<|P(\xi)| \leqslant H(P)^{-\omega}
$$

where $H(P)$ denotes the height of the polynomial $P(X)$, that is, the maximum of the moduli of its coefficients. Note that according to this definition we have $w_{1}(\xi)=\mu(\xi)-1$. Then, we set

$$
w(\xi)=\limsup _{n \rightarrow \infty} \frac{w_{n}(\xi)}{n} .
$$

According to Mahler's classification, we say that $\xi$ is:

- an $A$-number, if $w(\xi)=0$;

- an $S$-number, if $0<w(\xi)<+\infty$;

- a $T$-number, if $w(\xi)=+\infty$ and $w_{n}(\xi)<+\infty$ for every $n \geqslant 1$;

- a $U$-number, if $w_{n}(\xi)=+\infty$ for some $n \geqslant 1$.

In 1960, Wirsing [Wir60] proved that the class of $A$-numbers corresponds exactly to the set of real algebraic numbers. It is also known since Mahler [Mah32b] that the set of $S$-numbers has full Lebesgue measure. Thus, most real numbers have to be $S$-numbers. However, it is generally rather delicate to prove that a given number lies in this class. The following conjecture was suggested by Becker in his correspondence with Shallit in 1993. It claims that irrational automatic real numbers should behave in the same way as almost all real numbers with respect to Mahler's classification.

Conjecture 3.1. Automatic irrational real numbers are all $S$-numbers.

Since Liouville numbers are particular cases of $U$-numbers, this conjecture contains Theorem 2.1. A first step towards Conjecture 3.1 has recently been taken in [AB] and [ABL04]. Their result can be reformulated as follows. 


\section{B. AdAmCZewski and J. CASSAigne}

Theorem 3.2 (Adamczewski, Bugeaud and Luca [AB, ABL04]). The set of automatic irrational real numbers does not contain any $A$-number.

By combining ideas of the proof of Theorem 2.2 with Theorem 3.2 and a result of Baker [Bak64], we obtain the following result.

Theorem 3.3. Let $b \geqslant 2$ be an integer and $\mathbf{a}=\left(a_{n}\right)_{n \geqslant 0}$ be a non-eventually periodic automatic sequence. Let us assume that the internal sequence associated with a begins in an overlap (see $\S 4$ for a definition). Then, the automatic irrational real number

$$
\xi:=\sum_{n=0}^{+\infty} \frac{a_{n}}{b^{n}}
$$

is either an $S$-number or a $T$-number.

We end this section by mentioning that very special examples of Conjecture 3.1 are already known. Indeed, for any positive integer $d \geqslant 2$, the real number

$$
\xi:=\sum_{n=0}^{+\infty} \frac{1}{2^{d^{n}}}
$$

is an $S$-number, as follows from the work of Nishioka [Nis91].

\section{Finite automata, morphisms and Cobham's theorem}

Let $k \geqslant 2$ be an integer. An infinite sequence $\mathbf{a}=\left(a_{n}\right)_{n \geqslant 0}$ is said to be $k$-automatic if $a_{n}$ is a finitestate function of the base- $k$ representation of $n$. This means that there exists a finite automaton starting with the $k$-ary expansion of $n$ as input and producing the term $a_{n}$ as output. A nice reference on this topic is the book of Allouche and Shallit [AS03].

A more concrete definition of $k$-automatic sequences can be given as follows. Denote by $\Sigma_{k}$ the set $\{0,1, \ldots, k-1\}$. By definition, a $k$-automaton is a 6 -tuple

$$
A=\left(Q, \Sigma_{k}, \delta, q_{0}, \Delta, \tau\right),
$$

where $Q$ is a finite set of states, $\delta: Q \times \Sigma_{k} \rightarrow Q$ is the transition function, $q_{0}$ is the initial state, $\Delta$ is the output alphabet and $\tau: Q \rightarrow \Delta$ is the output function. For a state $q$ in $Q$ and for a finite word $W=w_{1} w_{2} \ldots w_{n}$ on the alphabet $\Sigma_{k}$, we define recursively $\delta(q, W)$ by $\delta(q, W)=$ $\delta\left(\delta\left(q, w_{1} w_{2} \ldots w_{n-1}\right), w_{n}\right)$. Let $n \geqslant 0$ be an integer and let $w_{r} w_{r-1} \ldots w_{1} w_{0}$ in $\left(\Sigma_{k}\right)^{r+1}$ be the $k$-ary expansion of $n$; thus, $n=\sum_{i=0}^{r} w_{i} k^{i}$. We denote by $W_{n}$ the word $w_{0} w_{1} \ldots w_{r}$. Then, a sequence $\mathbf{a}=\left(a_{n}\right)_{n \geqslant 0}$ is said to be $k$-automatic if there exists a $k$-automaton $A$ such that $a_{n}=\tau\left(\delta\left(q_{0}, W_{n}\right)\right)$ for all $n \geqslant 0$.

A classical example of a 2-automatic sequence is given by the Baum-Sweet sequence (see [BS76]) $\mathbf{a}=\left(a_{n}\right)_{n \geqslant 0}=110110010100100110010 \ldots$ This sequence is defined as follows: $a_{n}$ is equal to one if the binary expansion of $n$ contains no block of consecutive zeros of odd length, and zero otherwise. The Baum-Sweet sequence can be generated by the 2-automaton

$$
A=\left(\left\{q_{0}, q_{1}, q_{2}\right\},\{0,1\}, \delta, q_{0},\{0,1\}, \tau\right),
$$

where

$$
\begin{aligned}
& \delta\left(q_{0}, 0\right)=q_{1}, \delta\left(q_{0}, 1\right)=q_{0}, \quad \delta\left(q_{1}, 0\right)=q_{0}, \quad \delta\left(q_{1}, 1\right)=q_{2}, \\
& \delta\left(q_{2}, 0\right)=q_{2}, \quad \delta\left(q_{2}, 1\right)=q_{2},
\end{aligned}
$$

and $\tau\left(q_{0}\right)=\tau\left(q_{1}\right)=1, \tau\left(q_{2}\right)=0$ (see, for instance, [AS03]). 


\section{Diophantine PROPERTIES OF AUtOMATIC REAL NUMBERS}

\subsection{Morphisms and Cobham's theorem}

For a finite set $\mathcal{A}$, we denote by $\mathcal{A}^{*}$ the free monoid generated by $\mathcal{A}$. The empty word $\varepsilon$ is the neutral element of $\mathcal{A}^{*}$. Let $\mathcal{A}$ and $\mathcal{B}$ be two finite sets. A map from $\mathcal{A}$ to $\mathcal{B}^{*}$ can be uniquely extended to a homomorphism between the free monoids $\mathcal{A}^{*}$ and $\mathcal{B}^{*}$. We call morphism from $\mathcal{A}^{*}$ to $\mathcal{B}^{*}$ such a homomorphism. If there is a positive integer $k$ such that each element of $\mathcal{A}$ is mapped to a word of length $k$, then the morphism is called $k$-uniform or simply uniform. Similarly, a map from $\mathcal{A}$ to $\mathcal{B}$ can be uniquely extended to a homomorphism between the free monoids $\mathcal{A}^{*}$ and $\mathcal{B}^{*}$. Such a homomorphism is called a coding (the term 'letter-to-letter' morphism is also used in the literature). Thus, a coding is nothing else than a 1-uniform morphism.

A morphism $\sigma$ from $\mathcal{A}^{*}$ into itself is said to be prolongable if there exists a letter $a$ such that $\sigma(a)=a W$, where the word $W$ is such that $\sigma^{n}(W)$ is a non-empty word for every $n \geqslant 0$. In that case, the sequence of finite words $\left(\sigma^{n}(a)\right)_{n \geqslant 0}$ converges in $\mathcal{A}^{\infty}=\mathcal{A}^{*} \cup \mathcal{A}^{\mathbb{N}}$ endowed with its usual topology (see, for instance, [Lot02, ch. 2]) to an infinite word a denoted $\sigma^{\infty}(a)$. This infinite word is clearly a fixed point for $\sigma$ (extended by continuity to infinite words) and we say that $\mathbf{a}$ is generated by the morphism $\sigma$.

For instance, the Fibonacci morphism $\sigma_{1}$ defined over the alphabet $\{0,1\}$ by $\sigma_{1}(0)=01$ and $\sigma_{1}(1)=1$ is a non-uniform morphism which generates the celebrated Fibonacci infinite word

$$
\mathbf{a}=\lim _{n \rightarrow+\infty} \sigma_{1}^{n}(0)=010010100100101001 \ldots
$$

Uniform morphisms and automatic sequences are strongly connected, as the following result of Cobham [Cob72] shows. Theorem 4.1 in particular implies that finite automata produce sequences in real-time.

Theorem 4.1 (Cobham [Cob72]). A sequence is $k$-automatic if and only if it is the image under a coding of a fixed point of a $k$-uniform morphism.

Thus, one can always associate to a $k$-automatic sequence a a 5 -tuple $(\varphi, \sigma, i, \mathcal{A}, \mathcal{I})$, where $\sigma$ is a $k$-uniform morphism defined over a finite alphabet $\mathcal{I}, i$ is a letter of $\mathcal{I}, \varphi$ is a coding from $\mathcal{I}$ into $\mathcal{A}$, and such that

$$
\mathbf{a}=\varphi(\mathbf{i})
$$

with $\mathbf{i}=\sigma^{\infty}(i)$. The set $\mathcal{I}$ and the sequence $\mathbf{i}$ are respectively called the internal alphabet and the internal sequence associated to the 5 -tuple $(\varphi, \sigma, i, \mathcal{A}, \mathcal{I})$. With a slight abuse of language, we will say in the sequel that $\mathcal{I}$ (respectively $\mathbf{i}$ ) is the internal alphabet (respectively internal sequence) associated to a. Indeed, Cobham gives in fact a canonical way to associate with a a 5 -tuple $(\varphi, \sigma, i, \mathcal{A}, \mathcal{I})$.

We recall now that the $k$-kernel of a sequence $\mathbf{a}=\left(a_{n}\right)_{n \geqslant 0}$ is defined as the set $N_{k}(\mathbf{a})$ of all sequences $\left(a_{k^{i} \cdot n+j}\right)_{n \geqslant 0}$, where $i \geqslant 0$ and $0 \leqslant j<k^{i}$. This notion gives rise to another useful characterization of $k$-automatic sequences which was first proved in [Eil74].

ThEOREM 4.2 (Eilenberg [Eil74]). A sequence is $k$-automatic if and only if its $k$-kernel is finite.

\subsection{Words and repetitive patterns}

We end this section with some notation about repetitions in combinatorics on words. Let $\mathcal{A}$ be a finite set. The length of a word $W$ on the alphabet $\mathcal{A}$, that is, the number of letters composing $W$, is denoted by $|W|$. For any positive integer $\ell$, we write $W^{\ell}$ for the word $W \ldots W$ ( $\ell$ times repeated concatenation of the word $W$ ). More generally, for any positive real number $x$, we denote by $W^{x}$ the word $W^{\lfloor x\rfloor} W^{\prime}$, where $W^{\prime}$ is the prefix of $W$ of length $\lceil(x-\lfloor x\rfloor)|W|\rceil$. Here, and in all what follows, $\lfloor y\rfloor$ and $\lceil y\rceil$ denote, respectively, the integer part and the upper integer part of the real number $y$. 


\section{B. AdAmCZewski and J. CASSAigne}

An overlap is a word of the form $W W a$ where $W$ is a non-empty finite word and $a$ is the first letter of $W$.

\section{Proof of the main results}

This section is devoted to the proof of our main results, namely Theorems 2.2 and 3.3. Before proving Theorem 2.2, an auxiliary result that we state just below is needed. Roughly speaking, this result will allow us to control the repetitive patterns occurring as prefixes of any $k$-automatic sequence as a function of the size of its $k$-kernel. This result will also be used in the proof of Theorem 3.3.

Lemma 5.1. Let $\mathbf{u}$ be a non-eventually periodic $k$-automatic sequence defined on an alphabet $\mathcal{A}$. Let $U \in \mathcal{A}^{*}, V \in \mathcal{A}^{+}$and $s \in \mathbb{Q}$ be such that $U V^{s}$ is a prefix of the sequence $\mathbf{u}$. Let $m$ be the cardinality of the $k$-kernel of $\mathbf{u}$. Then,

$$
\frac{\left|U V^{s}\right|}{|U V|}<k^{m}
$$

Proof. Let $\mathbf{u}=\left(u_{n}\right)_{n \geqslant 0}$ be a non-eventually periodic $k$-automatic sequence defined on an alphabet $\mathcal{A}$. A triple $(h, p, l)$ of integers is said to be admissible (with respect to $\mathbf{u}$ ) if the following conditions hold:

(i) $1 \leqslant p \leqslant h \leqslant l$;

(ii) for all integers $n$ such that $h \leqslant n<l, u_{n-p}=u_{n}$;

(iii) $u_{l-p} \neq u_{l}$.

Let $m$ be the cardinality of the set $N_{k}(\mathbf{u})$. We shall prove that for every admissible triple $(h, p, l)$, $l<h k^{m}$. Let us assume, on the contrary, that $(h, p, l)$ is an admissible triple such that $l \geqslant h k^{m}$ and we aim at deriving a contradiction.

For $i$ ranging from zero to $m$, let us construct a triple $\left(h_{i}, p, l_{i}\right)$ admissible with respect to a sequence $\mathbf{u}^{(i)}$ of $N_{k}(\mathbf{u})$. We start with $\mathbf{u}^{(0)}=\mathbf{u}, h_{0}=h$ and $l_{0}=l$. Then, given $\mathbf{u}^{(i)}=\left(u_{n}^{(i)}\right)_{n \geqslant 0}$ and $\left(h_{i}, p, l_{i}\right)$, for $0 \leqslant i<m$, let $r_{i}$ be the remainder in the division of $l_{i}$ by $k$. We define $\mathbf{u}^{(i+1)}=$ $\left(u_{k n+r_{i}}^{(i)}\right)_{n \geqslant 0}$ by extracting from $\mathbf{u}^{(i)}$ letters at positions congruent to $r_{i}$ modulo $k$. Furthermore, we set $l_{i+1}=\left\lfloor l_{i} / k\right\rfloor$ and $h_{i+1}=l_{i+1}+p-\left\lfloor\left(l_{i}+p-h_{i}\right) / k\right\rfloor$.

We are now going to prove by induction on $i$ the following:
(a) $\mathbf{u}^{(i)} \in N_{k}(\mathbf{u})$;
(b) $h_{i} \leqslant h_{0}$;
(c) $l_{i} \geqslant h_{0} k^{m-i}$
(d) $\left(h_{i}, p, l_{i}\right)$ is admissible with respect to $\mathbf{u}^{(i)}$.

By assumption, these assertions are true for $i=0$. Let us assume that they hold for some $i$, $0 \leqslant i<m$, and let us prove that this also is the case for $i+1$.

(a) It is first clear that $\mathbf{u}^{(i)} \in N_{k}(\mathbf{u})$ implies that $\mathbf{u}^{(i+1)} \in N_{k}(\mathbf{u})$.

(b) We now prove the useful fact that $h_{i+1} \leqslant h_{i}$. Indeed, we have

$$
\begin{aligned}
h_{i}-h_{i+1} & =h_{i}-p-l_{i+1}+\left\lfloor\left(l_{i}+p-h_{i}\right) / k\right\rfloor \\
& =\left\lfloor\frac{k\left(h_{i}-p-l_{i+1}\right)+l_{i}+p-h_{i}}{k}\right\rfloor \\
& =\left\lfloor\frac{r_{i}+(k-1)\left(h_{i}-p\right)}{k}\right\rfloor \geqslant 0,
\end{aligned}
$$




\section{Diophantine PROPERTIES OF AUTOMATIC REAL NUMBERS}

since $r_{i} \geqslant 0, k \geqslant 2$ and, by assumption, $h_{i} \geqslant p$. Consequently, $h_{i+1} \leqslant h_{i} \leqslant h_{0}$ and assertion (b) holds.

(c) Since by assumption $l_{i} \geqslant h_{0} k^{m-i}$, we have that $l_{i+1}=\left\lfloor l_{i} / k\right\rfloor \geqslant\left\lfloor\left(k^{m-i} h_{0}\right) / k\right\rfloor=k^{m-i-1} h_{0}$.

(d) Now, let us prove that $\left(h_{i+1}, p, l_{i+1}\right)$ is admissible with respect to $\mathbf{u}^{(i+1)}$. As $p-h_{i} \leqslant 0$, $p-h_{i+1}=\left\lfloor\left(l_{i}+p-h_{i}\right) / k\right\rfloor-l_{i+1} \leqslant\left\lfloor l_{i} / k\right\rfloor-l_{i+1}=0$. Moreover, we get from assertion (c) that

$$
l_{i+1}-h_{i+1}=\left\lfloor\left(l_{i}+p-h_{i}\right) / k\right\rfloor-p \geqslant\left\lfloor\frac{h_{0} k^{m-i}+p-h_{i}}{k}\right\rfloor-p,
$$

and since $h_{i} \leqslant h_{0}$ by assertion (b) and $i<m$, we obtain

$$
\begin{aligned}
l_{i+1}-h_{i+1} & \geqslant\left\lfloor\frac{h_{i} k^{m-i}+p-h_{i}}{k}\right\rfloor-p=\left\lfloor\frac{h_{i}\left(k^{m-i}-1\right)-(k-1) p}{k}\right\rfloor \\
& \geqslant\left\lfloor\frac{\left(h_{i}-p\right)(k-1)}{k}\right\rfloor \geqslant 0 .
\end{aligned}
$$

We thus have

$$
1 \leqslant p \leqslant h_{i+1} \leqslant l_{i+1} .
$$

By assumption, we have $u_{n-p}^{(i)}=u_{n}^{(i)}$, for every integer $n$ such that $h_{i} \leqslant n<l_{i}$ and, thus,

$$
u_{n-j p}^{(i)}=u_{n}^{(i)},
$$

for all integers $n$ and $j$ such that $j \geqslant 0$ and $h_{i}+(j-1) p \leqslant n<l_{i}$. On the other hand, we get that

$$
k h_{i+1}+r_{i}=l_{i}+k p-k\left\lfloor\left(l_{i}+p-h_{i}\right) / k\right\rfloor \geqslant h_{i}+(k-1) p .
$$

Thus, $u_{n-k p}^{(i)}=u_{n}^{(i)}$, for every integer $n$ such that $k h_{i+1}+r_{i} \leqslant n<l_{i}$, and it a fortiori follows that $u_{k(n-p)+r_{i}}^{(i)}=u_{k n+r_{i}}^{(i)}$, for every integer $n$ such that $k h_{i+1}+r_{i} \leqslant k n+r_{i}<k l_{i+1}+r_{i}$. This implies that

$$
u_{n-p}^{(i+1)}=u_{n}^{(i+1)},
$$

for every integer $n$ such that $h_{i+1} \leqslant n<l_{i+1}$.

Since $k l_{i+1}+r_{i}=l_{i}$, we have $u_{l_{i+1}}^{(i+1)}=u_{l_{i}}^{(i)}$ and $u_{l_{i+1}-p}^{(i+1)}=u_{l_{i}-k p}^{(i)}$. Moreover, $l_{i+1} \geqslant h_{i+1}$ implies that $\left\lfloor\left(l_{i}+p-h_{i}\right) / k\right\rfloor \geqslant p$ and, thus, $l_{i} \geqslant h_{i}+(k-1) p$. Then, we obtain $l_{i}-p \geqslant h_{i}+(k-2) p$ and we infer from $(2)$ that

$$
u_{l_{i+1}-p}^{(i+1)}=u_{l_{i}-k p}^{(i)}=u_{\left(l_{i}-p\right)-(k-1) p}^{(i)}=u_{l_{i}-p}^{(i)} \neq u_{l_{i}}^{(i)}=u_{l_{i+1}}^{(i+1)} .
$$

Together with (1) and (3), this proves that the triple $\left(h_{i+1}, p, l_{i+1}\right)$ is admissible with respect to the sequence $\mathbf{u}^{(i+1)}$, hence assertion $(\mathrm{d})$.

We are now ready to end our proof by deriving a contradiction. Since $N_{k}(\mathbf{u})$ contains $m$ elements, at least two of the sequences $\mathbf{u}^{(i)}, 0 \leqslant i \leqslant m$, have to be the same. There thus exist two integers $i$ and $j, 0 \leqslant i<j \leqslant m$, such that $\left(h_{i}, p, l_{i}\right)$ and $\left(h_{j}, p, l_{j}\right)$ are two admissible triples for the same sequence. In particular, we have $u_{n}^{(i)}=u_{n}^{(j)}$ for any non-negative integer $n$. Moreover, $l_{j} \geqslant h_{0} k^{(m-j)} \geqslant h_{0} \geqslant h_{i}$, while $l_{j}=\left\lfloor l_{i} / k^{j-i}\right\rfloor<l_{i}$. Thus, $h_{i} \leqslant l_{j}<l_{i}$, and condition (ii) gives that $u_{l_{j}-p}^{(i)}=u_{l_{j}}^{(i)}$. On the other hand, condition (iii) implies that $u_{l_{j}-p}^{(i)}=u_{l_{j}-p}^{(j)} \neq u_{l_{j}}^{(j)}=u_{l_{j}}^{(i)}$, a contradiction. If the word $U V^{s}$ is a prefix of $\mathbf{u}$, as $\mathbf{u}$ is non-eventually periodic, there exists a maximal $s^{\prime} \in \mathbb{Q}, s^{\prime} \geqslant s$, such that $U V^{s^{\prime}}$ is a prefix of $\mathbf{u}$, and $\left(|U V|,|V|,\left|U V^{s^{\prime}}\right|\right)$ is an admissible triple. Therefore, $\left|U V^{s^{\prime}}\right|<|U V| k^{m}$ and, thus, $\left|U V^{s}\right|<|U V| k^{m}$ since $s^{\prime} \geqslant s$. This concludes the proof.

Here and after in this paper, we will consider rational numbers defined thanks to their $b$-adic expansion. Actually, we will often use the following construction: given two finite words $U$ and $V$ 


\section{B. AdAmCZewski and J. CASSAigne}

defined over the alphabet $\{0,1, \ldots, b-1\}$, we define the rational number

$$
p / q:=0 . U V V V \ldots V \ldots,
$$

having preperiod $U$ and period $V$ in its $b$-adic expansion. It may happen that $V=b-1$ and in such a case the $b$-adic expansion of $p / q$ is usually defined by $0 . a_{1} \ldots\left(a_{l}+1\right)$, where $U=a_{1} a_{2} \ldots a_{l}$. In the remainder of the paper, we allow inproper $b$-adic expansions of rational numbers, that is expansions ending with $(b-1) \ldots(b-1) \ldots$ So, when writing $p / q:=0 . a_{1} a_{2} \ldots a_{n} \ldots$, with $a_{n}$ ultimately equal to $b-1$, this will naturally mean $p / q=\sum_{n=0}^{+\infty} a_{n} / b^{n}$.

Before proving Theorem 2.2, we need to state the following result.

Lemma 5.2. Let $b \geqslant 2$ be an integer, $U$ and $V$ be two finite words defined over the alphabet $\{0,1, \ldots, b-1\}$ with length respectively equal to $r$ and $s$, and $p / q$ be a rational number with eventually periodic $b$-adic expansion

$$
\frac{p}{q}:=0 . a_{1} a_{2} \cdots=0 . U V V \ldots V \ldots
$$

Let $\xi:=0 . b_{1} b_{2} \ldots$ be a real number such that there exists a positive integer $j>r$ satisfying:

(i) $a_{n}=b_{n}$, for $1 \leqslant n<j$;

(ii) $a_{j} \neq b_{j}$.

Then,

$$
\left|\xi-\frac{p}{q}\right|>\frac{1}{b^{j+s}}
$$

Proof. We set $a_{j}=l$ and $b_{j}=m$. We first assume that $l>m$. Then, $l$ is a positive integer and we have

$$
\frac{p}{q}>0 . a_{1} a_{2} \ldots a_{j-1} l \underbrace{00 \ldots 0 \ldots 0}_{s-1 \text { times }} l,
$$

whereas

$$
\xi \leqslant 0 . a_{1} a_{2} \ldots a_{j-1}(m+1) \leqslant 0 . a_{1} a_{2} \ldots a_{j-1} l .
$$

This yields

$$
\frac{p}{q}-\xi>\frac{1}{b^{j+s}}
$$

Now, let us assume that $m>l$. Then, we have $l<b-1$ and

$$
\begin{aligned}
p / q<0 . a_{1} a_{2} \ldots a_{j-1} l \underbrace{(b-1)(b-1) \ldots(b-1)}_{s-1 \text { times }}(l+1) \\
\leqslant 0 . a_{1} a_{2} \ldots a_{j-1} l \underbrace{(b-1)(b-1) \ldots(b-1)}_{s \text { times }},
\end{aligned}
$$

whereas

$$
\xi \geqslant 0 . a_{1} a_{2} \ldots a_{j-1} m \geqslant 0 . a_{1} a_{2} \ldots a_{j-1}(l+1) .
$$

This gives

$$
\xi-\frac{p}{q}>\frac{1}{b^{j+s}}
$$

concluding the proof.

We are now ready to prove our main result. 


\section{Diophantine PROPERTIES OF AUTOMATIC REAL NUMBERS}

Proof of Theorem 2.2. Let $k$ and $b$ be two integers at least equal to two and let $\mathbf{a}=\left(a_{n}\right)_{n \geqslant 1}$ be an infinite sequence generated by a $k$-automaton and with values in $\{0,1, \ldots, b-1\}$. Let $\xi$ be the automatic real number defined by

$$
\xi:=\sum_{n=1}^{+\infty} \frac{a_{n}}{b^{n}} .
$$

Without loss of generality, we can assume that a is not eventually periodic. Indeed, otherwise $\xi$ would be a rational number and we would have $\mu(\xi)=1$.

Following Cobham's theorem (Theorem 4.1), there thus exist a $k$-uniform morphism $\sigma$ defined over a finite alphabet $\mathcal{I}$, a letter $i$ of $\mathcal{I}$ and a coding $\varphi$ from $\mathcal{I}$ into $\{0,1, \ldots, b-1\}$ such that

$$
\mathbf{a}=\varphi(\mathbf{i}),
$$

where $\mathbf{i}=\sigma^{\infty}(i)$. Let us also denote by $d$ the cardinality of the finite set $\mathcal{I}$ and by $m$ the cardinality of the $k$-kernel of the sequence $\mathbf{a}$.

Let $\delta$ be a positive number and let $(p, q)$ be a pair of positive integers. We shall prove that, for $q$ large enough, one always has

$$
\left|\xi-\frac{p}{q}\right| \geqslant \frac{1}{q^{M+\delta}}
$$

with $M=d k\left(k^{m}+1\right)$. Then, we will deduce the following upper bound for the irrationality measure of $\xi$ :

$$
\mu(\xi) \leqslant M
$$

as claimed in Theorem 2.2.

We are first going to introduce a sequence of rational numbers converging quite quickly to $\xi$ (see inequality (6)) and whose denominators do not grow too fast (see inequality (7)). These rational numbers are obtained thanks to Cobham's theorem and they will play a central role in this proof.

It follows from the pigeonhole principle that there is a letter $a$ that occurs at least twice in the prefix of length $d+1$ of the sequence $\mathbf{i}$. There thus exists a prefix of the sequence $\mathbf{i}$ of the form $U a V a$, where $U$ and $V$ are (possibly empty) finite words with $|U|+|V| \leqslant d-1$. Then, for any non-negative integer $n$, the finite word $\sigma^{n}(U a V a)$ is a prefix of the sequence $\mathbf{i}$. This implies that the finite word $\varphi\left(\sigma^{n}(U a V a)\right)$ is a prefix of the sequence a. Let $U_{n}=\varphi\left(\sigma^{n}(U)\right)$ and $V_{n}=\varphi\left(\sigma^{n}(a V)\right)$. We set $r_{n}=\left|U_{n}\right|, s_{n}=\left|V_{n}\right|$, and $t_{n}=\left|\varphi\left(\sigma^{n}(a)\right)\right|$. Since $\sigma$ is a $k$-uniform morphism and $\varphi$ is a coding, we get $r_{n}=k^{n}|U|, s_{n}=k^{n}|a V|$ and $t_{n}=k^{n}$. We also set $q_{n}=b^{r_{n}}\left(b^{s_{n}}-1\right)$. An easy computation shows that there exists a positive integer $p_{n}$ such that the rational number $p_{n} / q_{n}$ has the following eventually periodic $b$-adic expansion:

$$
\frac{p_{n}}{q_{n}}:=0 . U_{n} V_{n} V_{n} V_{n} \ldots V_{n} \ldots
$$

or, in short,

$$
\frac{p_{n}}{q_{n}}=0 . U_{n} \overline{V_{n}}
$$

Since the word $\varphi\left(\sigma^{n}(U a V a)\right)$ is a common prefix of the sequence a and of $U_{n} \overline{V_{n}}$, it follows that

$$
\left|\xi-\frac{p_{n}}{q_{n}}\right|<\frac{1}{b^{r_{n}+s_{n}+t_{n}}} .
$$

Moreover,

$$
\frac{t_{n}}{r_{n}+s_{n}}=\frac{k^{n}}{k^{n}|U a V|}=\frac{1}{|U a V|} \geqslant \frac{1}{d} .
$$




\section{B. Adamczewski and J. Cassaigne}

This implies that

$$
\left|\xi-\frac{p_{n}}{q_{n}}\right|<\frac{1}{q_{n}^{1+1 / d}}
$$

On the other hand, $r_{n+1}=k r_{n}$ and $s_{n+1}=k s_{n}$. This implies that

$$
q_{n+1}=b^{k r_{n}}\left(b^{k s_{n}}-1\right)<\left(b^{r_{n}}\left(b^{s_{n}}-1\right)\right)^{k+\varepsilon}=q_{n}^{k+\varepsilon},
$$

for any positive real number $\varepsilon$ and any integer $n$ large enough. Now, we fix a positive real number $\varepsilon$ that we choose small enough to ensure that

$$
d \varepsilon+d \varepsilon^{2}+\varepsilon d k^{m}+\varepsilon d k<\delta / 2 .
$$

Let $n_{0}$ be an integer such that (7) holds for any integer $n \geqslant n_{0}$.

We now have to remark that Lemma 5.1 naturally gives rise to an upper bound for the approximation of $\xi$ by $p_{n} / q_{n}$. By Lemma 5.1, if $U_{n} V_{n}^{s}$ is a prefix of the sequence a, then $\left|U_{n} V_{n}^{s}\right|<$ $\left|U_{n} V_{n}\right| k^{m}=\left(r_{n}+s_{n}\right) k^{m}$. We deduce that $\xi$ and $p_{n} / q_{n}$ cannot have the same first $\left|U_{n} V_{n}\right| k^{m}$ digits. Following (5), the conditions of Lemma 5.2 are thus fulfilled with $r_{n}+s_{n}+t_{n}<j \leqslant\left(r_{n}+s_{n}\right) k^{m}$. Consequently, we get that

$$
\left|\xi-\frac{p_{n}}{q_{n}}\right| \geqslant \frac{1}{b^{\left(r_{n}+s_{n}\right) k^{m}+s_{n}}}>\frac{1}{\left(b^{r_{n}}\left(b^{s_{n}}-1\right)\right)^{k^{m}+1+\varepsilon}}=\frac{1}{q_{n}^{k^{m}+1+\varepsilon}},
$$

for $n$ large enough. There thus exists a positive integer $n_{1}, n_{1} \geqslant n_{0}$, such that

$$
\left|\xi-\frac{p_{n}}{q_{n}}\right|>\frac{1}{q_{n}^{k^{m}+1+\varepsilon}}
$$

for any $n \geqslant n_{1}$.

We are now ready for the last step of the proof. Let us consider a rational number $p / q$ with $q$ large enough and let us prove that inequality (4) holds. Let us assume that $q$ satisfies the following three inequalities: $2 q \geqslant q_{n_{1}+1}^{1 / d}, q \geqslant 2^{1+2 M / \delta}$ and $q \geqslant 2^{1+d k+\delta / 2}$. Moreover, let us assume that

$$
\left|\xi-\frac{p}{q}\right|<\frac{1}{(2 q)^{1+d(k+\varepsilon)}} \text {. }
$$

First, note that if (10) is not satisfied, then (4) holds. Indeed, let us assume that (10) is not satisfied by the rational $p / q$. We have $d k^{m+1}>2$ and (8) implies that $d \varepsilon<\delta / 2$. We thus derive that $2+d(k+\varepsilon)<M+\delta$, and since $q \geqslant 2^{1+d k+\delta / 2}>2^{1+d k+d \varepsilon}$, we have

$$
(2 q)^{1+d(k+\varepsilon)}<q^{2+d(k+\varepsilon)}<q^{M+\delta} .
$$

This ensures that inequality (4) is satisfied by the rational $p / q$.

Now, since by assumption $2 q \geqslant q_{n_{1}+1}^{1 / d}$, it follows from (7) that there exists a unique positive integer $n_{2}>n_{1} \geqslant n_{0}$ such that

$$
q_{n_{2}-1} \leqslant(2 q)^{d}<q_{n_{2}}<q_{n_{2}-1}^{k+\varepsilon} .
$$

The triangle inequality gives

$$
\left|\xi-\frac{p_{n_{2}}}{q_{n_{2}}}\right| \geqslant|| \xi-\frac{p}{q}|-| \frac{p_{n_{2}}}{q_{n_{2}}}-\frac{p}{q}|| .
$$

If $p / q$ and $p_{n_{2}} / q_{n_{2}}$ are two distinct rationals, we obviously get that

$$
\left|\frac{p_{n_{2}}}{q_{n_{2}}}-\frac{p}{q}\right| \geqslant \frac{1}{q q_{n_{2}}}
$$




\section{Diophantine PROPERTIES OF AUTOMATIC REAL NUMBERS}

Moreover, $q_{n_{2}} \leqslant q_{n_{2}-1}^{k+\varepsilon} \leqslant(2 q)^{d(k+\varepsilon)}$ and we thus derive from the previous inequality that

$$
\left|\frac{p_{n_{2}}}{q_{n_{2}}}-\frac{p}{q}\right| \geqslant \frac{2}{(2 q)^{1+d(k+\varepsilon)}}>2\left|\xi-\frac{p}{q}\right| .
$$

Then, (10) and (12) imply that, if $p / q \neq p_{n_{2}} / q_{n_{2}}$,

$$
\left|\xi-\frac{p_{n_{2}}}{q_{n_{2}}}\right|>\frac{1}{2 q q_{n_{2}}} .
$$

Now, we infer from (11) that $2 q<q_{n_{2}}^{1 / d}$. This gives

$$
\left|\xi-\frac{p_{n_{2}}}{q_{n_{2}}}\right|>\frac{1}{q_{n_{2}}^{1+1 / d}},
$$

which is a contradiction with (6). It thus follows that, under the assumption (10), we necessarily have $p / q=p_{n_{2}} / q_{n_{2}}$. In that case, since $n_{2} \geqslant n_{1}$, we infer from (9) that

$$
\left|\xi-\frac{p}{q}\right|=\left|\xi-\frac{p_{n_{2}}}{q_{n_{2}}}\right| \geqslant \frac{1}{q_{n_{2}}^{k^{m}+1+\varepsilon}}
$$

and since $(2 q)^{d(k+\varepsilon)} \geqslant q_{n_{2}}$, we obtain

$$
\left|\xi-\frac{p}{q}\right| \geqslant \frac{1}{(2 q)^{d(k+\varepsilon)\left(k^{m}+1+\varepsilon\right)}} .
$$

We infer from (8) and from the assumption that $q \geqslant 2^{1+2 M / \delta}$ that

$$
(2 q)^{d(k+\varepsilon)\left(k^{m}+1+\varepsilon\right)} \leqslant(2 q)^{M+\delta / 2} \leqslant q^{M+\delta / 2} 2^{M+\delta / 2} \leqslant q^{M+\delta}
$$

and this implies that

$$
\left|\xi-\frac{p}{q}\right| \geqslant \frac{1}{q^{M+\delta}}
$$

Inequality (4) thus holds for any

$$
q \geqslant \max \left\{\frac{q_{n_{1}+1}^{1 / d}}{2}, 2^{1+2 M / \delta}, 2^{1+d k+\delta / 2}\right\} .
$$

This concludes the proof.

We end this section by proving Theorem 3.3. The proof follows the same lines as that of Theorem 2.2. The key point is a general criterion to prove that a real number is not a $U$-number. This result is due to Baker [Bak64] and we recall it now.

Theorem 5.3 (Baker [Bak64]). Let $\xi$ be a real number, $\varepsilon$ be a positive number and $\left(p_{n} / q_{n}\right)_{n \geqslant 1}$ be a sequence of distinct rational numbers. Let us assume that the following conditions hold:

$$
\left|\xi-\frac{p_{n}}{q_{n}}\right|<\frac{1}{q_{n}^{2+\varepsilon}}
$$

and

Then, $\xi$ is not a $U$-number.

$$
\limsup _{n \rightarrow \infty} \frac{\log \left(q_{n+1}\right)}{\log \left(q_{n}\right)}<+\infty
$$

Proof of Theorem 3.3. We keep the notation of Theorem 2.2. Let us assume that the sequence $\mathbf{i}$ begins in an overlap $U U a$, where $U$ is a finite word and $a$ is the first letter of $U$. Then, for any nonnegative integer $n$, the finite word $\varphi\left(\sigma^{n}(U U a)\right)$ is a prefix of the sequence a. Let $s_{n}=\left|\varphi\left(\sigma^{n}(U)\right)\right|$. 


\section{B. Adamczewski and J. Cassaigne}

We also set $q_{n}=b^{s_{n}}-1$. There thus exists a positive integer $p_{n}$ such that the rational number $p_{n} / q_{n}$ has the following periodic $b$-adic expansion:

$$
\frac{p_{n}}{q_{n}}=0 . \overline{\varphi\left(\sigma^{n}(U)\right)}
$$

Since the word $\varphi\left(\sigma^{n}(U U a)\right)$ is a prefix of the sequence a, it follows that

$$
\left|\xi-\frac{p_{n}}{q_{n}}\right|<\frac{1}{b^{2 s_{n}+t_{n}}}
$$

where $t_{n}=\left|\varphi\left(\sigma^{n}(a)\right)\right|$. Moreover, since $\sigma$ is a $k$-uniform morphism, $s_{n}=k^{n}|U|$ and $t_{n}=k^{n}$, and we get that

$$
\left|\xi-\frac{p_{n}}{q_{n}}\right|<\frac{1}{q_{n}^{2+\varepsilon}}
$$

where $\varepsilon=1 /|U|$. On the other hand, the definition of $q_{n}$ implies that

$$
\limsup _{n \rightarrow \infty} \frac{\log \left(q_{n+r}\right)}{\log \left(q_{n}\right)}=k^{r}<+\infty
$$

for any positive integer $r$. We now infer from Lemmas 5.1 and 5.2 that

$$
\left|\xi-\frac{p_{n}}{q_{n}}\right|>\frac{1}{b^{s_{n}\left(k^{m}+1\right)}} .
$$

There thus exists a positive integer $r$ large enough to ensure that the sequence $\left(p_{r n} / q_{r n}\right)_{n \geqslant 0}$ consists of distinct rational numbers. Indeed, let $n^{\prime}>n \geqslant 0$ be two integers such that $p_{n} / q_{n}=p_{n^{\prime}} / q_{n^{\prime}}$. It follows from (13) and (16) that $2 s_{n^{\prime}}+t_{n^{\prime}}<s_{n}\left(k^{m}+1\right)$, which gives $2 k^{n^{\prime}}|U|+k^{n^{\prime}}<k^{n}|U|\left(k^{m}+1\right)$. We thus have

$$
k^{n^{\prime}-n}<\frac{|U|\left(k^{m}+1\right)}{2|U|+1}<k^{m}
$$

and $n^{\prime}<n+m$.

Then, (14) and (15) show that the conditions of Theorem 5.3 are fulfilled. This implies that $\xi$ is not a $U$-number. On the other hand, we infer from Theorem 3.2 that $\xi$ is not an $A$-number. This ends the proof.

\section{An emblematic example: the Thue-Morse-Mahler numbers}

In Theorem 2.2, we have obtained a general upper bound for the irrationality measure of real numbers generated by finite automata. It turns out that for a specific automatic real number the method introduced in the proof of Theorem 2.2 will likely give rise to a better estimate. In the present section, we choose to illustrate this general idea by considering the Thue-Morse-Mahler numbers as a particular example.

For an integer $b$ at least equal to two, we define the $b$-adic Thue-Morse-Mahler number $\xi_{b}$ by

$$
\xi_{b}=\sum_{k=0}^{+\infty} \frac{a_{k}}{b^{k}},
$$

where the sequence $\mathbf{a}=\left(a_{n}\right)_{n \geqslant 0}$ is the Thue-Morse sequence. This sequence is defined as follows: $a_{n}$ is equal to zero (respectively to one) if the sum of the digits in the binary expansion of $n$ is even (respectively is odd). Thus, the $b$-adic expansion of $\xi_{b}$ begins with $0.110100110010 \ldots$ These numbers were first considered by Mahler who proved that they are transcendental in [Mah29] (see also [Dek77]). 


\section{Diophantine PROPERTIES OF AUTOMATIC REAL NUMBERS}

It is easy to check that the Thue-Morse sequence can be generated by the 2-automaton

$$
A=\left(\left\{q_{0}, q_{1}\right\},\{0,1\}, \delta, q_{0},\{0,1\}, \tau\right),
$$

where

$$
\delta\left(q_{0}, 0\right)=\delta\left(q_{1}, 1\right)=q_{0}, \quad \delta\left(q_{0}, 1\right)=\delta\left(q_{1}, 0\right)=q_{1},
$$

and $\tau\left(q_{0}\right)=0, \tau\left(q_{1}\right)=1$. It is also well-known, as a consequence of Theorem 4.1, that this sequence is the fixed point beginning with zero of the following binary 2-uniform morphism:

$$
\begin{array}{ccc} 
& \sigma_{2} & \\
0 & \longmapsto & 01 \\
1 & \longmapsto & 10
\end{array}
$$

Theorem 2.2 thus implies that the irrationality measure of $\xi_{b}$ satisfies $\mu\left(\xi_{b}\right) \leqslant 20$ (independently of the integer $b$ ), since in that case $d=k=m=2$ (the fact that $m=2$ is, for instance, proved in [AS03]). However, a better use of the method described in the previous section leads to the following improvement.

Theorem 6.1. For any $b \geqslant 2$, we have

$$
\mu\left(\xi_{b}\right) \leqslant 5 .
$$

Proof. From now on, we fix a positive integer $b \geqslant 2$ and we set $\xi:=\xi_{b} / b$. Thus,

$$
\xi=0 . a_{0} a_{1} a_{2} a_{3} \ldots
$$

It is obvious that $\xi$ and $\xi_{b}$ have the same irrationality measure. Let $\delta$ be a positive number. We want to prove that the inequality

$$
\left|\xi-\frac{p}{q}\right|<\frac{1}{q^{5+\delta}}
$$

has only finitely many solutions $(p, q) \in \mathbb{Z}^{2}$.

As in the proof of Theorem 2.2, we are first going to introduce an infinite sequence of rationals converging to $\xi$. We can remark that $\sigma_{2}^{3}(0)=01101001$ and thus the sequence a begins in the word 011010. More generally, it follows that a begins in $\sigma_{2}^{n}(011010)$ for any non-negative integer $n$. Since 0110 is a prefix of $\sigma_{2}^{n}(0)$ for $n \geqslant 2$, we deduce that the word

$$
\sigma_{2}^{n}(011) \sigma_{2}^{n}(01) 0110=\left(\sigma_{2}^{n}(011)^{1+2 / 3}\right) 0110
$$

is a prefix of a for $n \geqslant 2$. Now, we set $q_{n}=\left(b^{3 \cdot 2^{n}}-1\right)$. It follows from an easy computation that there exists a positive integer $p_{n}$ such that the rational number $p_{n} / q_{n}$ has the following periodic $b$-adic expansion:

$$
\frac{p_{n}}{q_{n}}:=0 . \sigma_{2}^{n}(011) \sigma_{2}^{n}(011) \ldots \sigma_{2}^{n}(011) \ldots
$$

or, in short,

$$
\frac{p_{n}}{q_{n}}=0 . \overline{\sigma_{2}^{n}(011)}
$$

The $b$-adic expansion of $p_{n} / q_{n}$ begins with

$$
\sigma_{2}^{n}(011) \sigma_{2}^{n}(011)=\sigma_{2}^{n}(011) \sigma_{2}^{n}(01) \sigma_{2}^{n}(1)
$$

and we deduce that it begins with

$$
\sigma_{2}^{n}(011) \sigma_{2}^{n}(01) 1001
$$

when $n \geqslant 2$. Since $\sigma_{2}$ is a 2-uniform morphism, we easily check that $\left|\sigma_{2}^{n}(W)\right|=2^{n}|W|$ for any finite word $W$. We thus infer from (18) and (19) that the first $\left(5 \cdot 2^{n}\right)$ th digits in the $b$-adic expansion of 


\section{B. Adamczewski and J. Cassaigne}

$p_{n} / q_{n}$ and of $\xi$ are the same while the following four digits are, respectively, 1001 and 0110 . This implies that

$$
\frac{1}{b^{5 \cdot 2^{n}+3}} \leqslant\left|\xi-\frac{p_{n}}{q_{n}}\right|<\frac{1}{b^{5 \cdot 2^{n}+2}} .
$$

Let $\varepsilon$ be a positive number chosen small enough to ensure that

$$
(2 q)^{4+3 \varepsilon / 2}<(2 q)^{5+11 \varepsilon / 2+3 \varepsilon^{2} / 2}<q^{5+\delta}
$$

for any integer $q$ large enough, say for any $q \geqslant M>2$. The definition of $q_{n}$ implies that there exists a positive integer $n_{0}$ such that

$$
\frac{1}{q_{n}^{1+2 / 3+\varepsilon}} \leqslant\left|\xi-\frac{p_{n}}{q_{n}}\right|<\frac{1}{q_{n}^{1+2 / 3}}
$$

for any $n \geqslant n_{0}$. On the other hand, we also have

$$
q_{n+1}=\left(b^{3 \cdot 2^{n+1}}-1\right)<q_{n}^{2+\varepsilon}
$$

for any integer $n$ large enough, say $n \geqslant n_{1} \geqslant n_{0}$.

Let us assume that there exists a rational number $p / q$ satisfying inequality (17) and such that the following two inequalities hold: $2 q \geqslant q_{n_{1}}^{2 / 3}$ and $q \geqslant M$. We now aim at deriving a contradiction.

Since by assumption $2 q \geqslant q_{n_{1}}^{2 / 3}$, it follows from (22) that there exists a unique positive integer $n_{2}>n_{1}$ such that

$$
q_{n_{2}-1} \leqslant(2 q)^{3 / 2}<q_{n_{2}}<q_{n_{2}-1}^{2+\varepsilon} \leqslant(2 q)^{3 / 2(2+\varepsilon)} .
$$

The triangle inequality gives

$$
\left|\xi-\frac{p_{n_{2}}}{q_{n_{2}}}\right| \geqslant|| \xi-\frac{p}{q}|-| \frac{p_{n_{2}}}{q_{n_{2}}}-\frac{p}{q}|| .
$$

Then, we infer from (17), (21) and (23) that $p / q$ and $p_{n_{2}} / q_{n_{2}}$ are two distinct rationals. Otherwise we would have from (21) that

$$
\left|\xi-\frac{p}{q}\right|=\left|\xi-\frac{p_{n_{2}}}{q_{n_{2}}}\right| \geqslant \frac{1}{q_{n_{2}}^{1+2 / 3+\varepsilon}}
$$

and then (23) would give

$$
\left|\xi-\frac{p}{q}\right| \geqslant \frac{1}{(2 q)^{3 / 2(2+\varepsilon)(1+2 / 3+\varepsilon)}}=\frac{1}{(2 q)^{5+11 \varepsilon / 2+3 \varepsilon^{2} / 2}} .
$$

In such a case, (20) would give

$$
\left|\xi-\frac{p}{q}\right| \geqslant \frac{1}{q^{5+\delta}}
$$

and we would reach a contradiction with (17). Thus, $p / q$ and $p_{n_{2}} / q_{n_{2}}$ are distinct and we immediately get that

$$
\left|\frac{p_{n_{2}}}{q_{n_{2}}}-\frac{p}{q}\right| \geqslant \frac{1}{q q_{n_{2}}} .
$$

We then derive, using (23), (20) and (17), that

$$
\left|\frac{p_{n_{2}}}{q_{n_{2}}}-\frac{p}{q}\right| \geqslant \frac{2}{(2 q)^{1+3 / 2(2+\varepsilon)}}=\frac{2}{(2 q)^{4+3 \varepsilon / 2}}>\frac{2}{q^{5+\delta}}>2\left|\xi-\frac{p}{q}\right| .
$$

Then, (17) and (24) imply that

$$
\left|\xi-\frac{p_{n_{2}}}{q_{n_{2}}}\right|>\frac{1}{2 q q_{n_{2}}}
$$




\section{Diophantine PROPERTIES OF AUTOMATIC REAL NUMBERS}

Now, we infer from $(23)$ that $2 q<q_{n_{2}}^{2 / 3}$. This gives

$$
\left|\xi-\frac{p_{n_{2}}}{q_{n_{2}}}\right|>\frac{1}{q_{n_{2}}^{1+2 / 3}},
$$

which is a contradiction with (21). Thus, any rational $p / q$ satisfying inequality (17) has a denominator $q$ at most equal to $\max \left\{M, q_{n_{1}}^{2 / 3} / 2\right\}$. This ends the proof.

\section{Some generalizations and concluding remarks}

A first possibility of extension of the present approach is to focus on the representations of real numbers in algebraic bases, that is, to replace the integer $b$ with an algebraic real number $\beta$ greater than one. We can consider both the analog of the Loxton-van der Poorten conjecture and of the Becker conjecture, the number field $\mathbb{Q}(\beta)$ taking the place of the field of rationals. For an algebraic real number $\beta$ and an automatic sequence $\left(a_{n}\right)_{n \geqslant 0}$ with values in $\{0,1, \ldots,\lfloor\beta\rfloor\}$, it is thus likely that the real number

$$
\xi:=\sum_{n \geqslant 0} \frac{a_{n}}{\beta^{n}}
$$

either lies in $\mathbb{Q}(\beta)$ or is transcendental (this is the analog of the Loxton-van der Poorten conjecture), and is always an $S$-number in the latter case (this is the analog of Becker's conjecture).

Actually, if we restrict our attention to the $\beta$-expansions introduced by Rényi [Ren57] and on a Pisot or a Salem base, the analog of the Loxton-van der Poorten conjecture is proved in [AB06b] following the approach of $[\mathrm{AB}]$. Under the same restriction, our method is sufficient, without introducing new ideas, to prove the following result. We recall that a Pisot (respectively a Salem) number is a real algebraic integer, greater than one, whose complex conjugates lie inside the open unit disc (respectively inside the closed unit disc, with at least one of them on the unit circle).

Theorem 7.1. Let $\beta$ be a Pisot or a Salem number. Then, the $\beta$-expansion of a Liouville number cannot be generated by a finite automaton.

Moreover, we could provide an explicit upper bound for the irrationality measure of $\xi$. Note also that in [Bak64] Baker proved an analog of Theorem 5.3 for number fields. This yields the following result. In the sequel, we will denote by $d_{\beta}(x)$ the $\beta$-expansion of the real number $x$.

Theorem 7.2. Let $\beta$ be a Pisot or a Salem number, and $\xi$ be a real number that does not lie in $\mathbb{Q}(\beta)$, and such that

$$
d_{\beta}(\xi)=a_{0} \cdot a_{1} a_{2} \ldots a_{n} \ldots,
$$

where $\mathbf{a}=\left(a_{n}\right)_{n \geqslant 0}$ is an automatic sequence. Let us assume, moreover, that the internal sequence associated with a begins in an overlap (see $\S 4$ for a definition). Then, $\xi$ is either a $S$-number or a T-number.

However, new ideas are really needed for dealing with an arbitrary algebraic real number $\beta$. Indeed, in the general case the approximants $\alpha_{n}$ provided by an automatic $\beta$-expansion (see $\S 8$ ) are such that the inequality

$$
\left|\xi-\alpha_{n}\right| \ll H\left(\alpha_{n}\right)^{-1-\varepsilon}
$$

does not necessarily hold. We recall that the height $H(\alpha)$ of an algebraic number $\alpha$ is defined as the height of its minimal polynomial (see $\S 3$ for a definition). Another difficulty also appears if we want to replace the Rényi $\beta$-expansion by other representations in base $\beta$ (such as, for instance, that arising from the lazy algorithm). Indeed, it is not clear how to obtain the analog of Lemma 5.2 for such representations. 


\section{B. Adamczewsiki and J. Cassaigne}

We end this section with a digression on $U$-numbers and some other generalizations of the approach introduced in this paper. As was remarked by LeVeque [Lev53], the class of $U$-numbers can be divided into infinitely many subclasses of interest, each of them corresponding to the approximation by algebraic numbers whose degree is bounded by a fixed positive integer. The simplest subclass, the class of $U_{1}$-numbers, exactly corresponds to the Liouville numbers. Given a positive integer $m$, a real number $\xi$ is more generally called a $U_{m}$-number if

$$
w_{m}(\xi)=+\infty \text { and } w_{n}(\xi)<+\infty, \text { for } 0<n<m \text {. }
$$

Thus, the set of $U$-numbers is exactly the infinite union on all of the positive integers $n$ of the sets formed by the $U_{n}$-numbers. We refer the reader to $\S 3$ for a definition of $w_{n}(\xi)$.

Another possible generalization of the present work consists of replacing the $b$-adic expansion with the continued fraction expansion of real numbers. Then, this naturally leads one to consider the notion of automatic continued fraction, that is, to consider real numbers whose continued fraction expansion can be generated by a finite automaton. These real numbers were studied by several authors (see, for instance, [AB05] and the references therein). In particular, it is believed but not yet proved that such numbers are either quadratic or transcendental. This corresponds, in this framework, to the analog of the Loxton-van der Poorten conjecture. In this way, Becker's conjecture can be translated as follows: non-quadratic automatic continued fractions are all $S$-numbers. Note that it is obvious that no Liouville number can have an automatic continued fraction expansion, since the latter numbers, by definition, have bounded partial quotients. Thus, the analog to Theorem 2.1 would be that the set of automatic continued fractions does not contain any $U_{2}$-numbers. The method introduced in this paper is sufficient to prove a first step toward such a result and would even, in this particular case, provide an explicit upper bound for $w_{2}(\xi)$.

THEOREM 7.3. Let $\mathbf{a}=\left(a_{n}\right)_{n \geqslant 0}$ be an automatic sequence of positive integers. Let us assume that the first letter of the internal sequence associated with a appears at least twice (see $\S 4$ for a definition). Then, the automatic continued fraction

$$
\xi:=\left[a_{0}, a_{1}, a_{2}, \ldots\right]
$$

is not a $U_{2}$-number.

As an example, let us consider a uniform morphism $\sigma$ defined from the monoid $\{a, b\}^{*}$ into itself, where $a$ and $b$ are two distinct positive integers. Let us assume that the sequence $\mathbf{a}=$ $\left(a_{n}\right)_{n \geqslant 0}$ is a non-eventually periodic fixed point for $\sigma$. Then, the real number $\xi:=\left[a_{0}, a_{1}, a_{2}, \ldots\right]$ is transcendental (this is proved in [AB05]) and is not a $U_{2}$-number as a consequence of Theorem 7.3.

Note that it could also be interesting to consider continued fractions associated with regular sequences as introduced by Allouche and Shallit [AS92]. These sequences provide a natural generalization of automatic sequences for sequences with values lying in an infinite set.

\section{Outlines of the proof of Theorems 7.1, 7.2, and 7.3}

We begin with the main steps for proving Theorems 7.1 and 7.2. From now on, $\beta$ denotes a Pisot or a Salem number of degree $l$. Let $\xi$ be a real number such that

$$
d_{\beta}(\xi)=0 . a_{1} a_{2} \ldots a_{n} \ldots,
$$

where $\mathbf{a}=\left(a_{n}\right)_{n \geqslant 1}$ is an automatic sequence. Note that if the sequence $\mathbf{a}$ is eventually periodic, then $\xi$ belongs to $\mathbb{Q}(\beta)$ and cannot be a Liouville number. We keep the notation of the proof of Theorem 2.2. Then, we first replace the sequence of rational approximants $\left(p_{n} / q_{n}\right)_{n \geqslant 0}$ with a sequence of algebraic numbers $\left(\alpha_{n}\right)_{n \geqslant 0}$ lying in the same number field $\mathbb{Q}(\beta)$. We set

$$
\alpha_{n}:=0 . U_{n} \overline{V_{n}},
$$




\section{Diophantine PROPERTIES OF AUtOMATIC REAL NUMBERS}

where $0 . U_{n} \overline{V_{n}}$ is a possibly inproper expansion in base $\beta$. There thus exists an integer polynomial $P_{n}(X)$ of degree at most $r_{n}+s_{n}$ and such that

$$
\alpha_{n}=\frac{P_{n}(\beta)}{\beta^{r_{n}}\left(\beta^{s_{n}}-1\right)} .
$$

Since $\beta$ is a Pisot or a Salem number of degree $l$, one can use the product formula and the associated notion of height to easily derive that $H\left(\alpha_{n}\right) \ll\left(r_{n}+s_{n}\right)^{l-1} \beta^{r_{n}+s_{n}}$ (see, for instance, [Ada04] where similar arguments are given). Moreover, we have

$$
\left|\xi-\alpha_{n}\right| \ll \frac{1}{\beta^{r_{n}+s_{n}+t_{n}}},
$$

and, thus, for every positive $\varepsilon$, we obtain that

$$
\left|\xi-\alpha_{n}\right| \ll \frac{1}{H\left(\alpha_{n}\right)^{1+1 / d-\varepsilon}} .
$$

We now want to apply a classical trick from Diophantine approximation, as stated in Lemma 8.1 (see for instance [Bug04, Gut68, Lev53] for more details). The proof of this result essentially relies on the following Liouville type inequality (see, for instance, [Bug04, p. 227]). Let $\alpha$ and $\alpha^{\prime}$ be two distinct algebraic numbers of degree, respectively, equal to $i$ and $j$. Then, there exists a constant $c$ only depending on $i$ and $j$ such that

$$
\left|\alpha-\alpha^{\prime}\right|>c H(\alpha)^{-j} H\left(\alpha^{\prime}\right)^{-i} .
$$

Lemma 8.1. Let $\xi$ be a real number. Let us assume that there exists an infinite sequence $\left(\alpha_{n}\right)_{n \geqslant 1}$ of algebraic numbers of degree $r$ such that:

(i) $H\left(\alpha_{n}\right)<H\left(\alpha_{n+1}\right)<H\left(\alpha_{n}\right)^{s}$;

(ii) $H\left(\alpha_{n}\right)^{-\eta^{\prime}}<\left|\xi-\alpha_{n}\right|<H\left(\alpha_{n}\right)^{-\eta}$.

Then, $\xi$ is not a $U_{t}$-number for any integer $t<\eta$.

Here, we can choose $\varepsilon$ small enough in (25) to ensure that $\eta=1+1 / d-\varepsilon>1$, and we want to prove that $\xi$ is not a Liouville number or, equivalently, that $\xi$ is not a $U_{1}$-number. It thus remains to prove that the sequence $\left(\alpha_{n}\right)_{n \geqslant 0}$ enjoys the two following properties:

- $\alpha_{n}$ is not a too good approximation to $\xi$; this corresponds to the left-hand side of assumption (ii);

- there exists a subsequence of $\left(\alpha_{n}\right)_{n \geqslant 0}$ satisfying assumption (i).

As in the proof of Theorem 2.2, both properties are ensured thanks to Lemma 5.1 and the following analog of Lemma 5.2. Since this is not a classical result and some difficulties appear, we choose to give below a complete proof of Lemma 8.2.

Lemma 8.2. Let $\beta>1$ be a Pisot or a Salem number of degree $l$. Let $U$ and $V$ be two finite words defined over the alphabet $\{0,1, \ldots,\lfloor\beta\rfloor\}$ with length respectively equal to $r$ and $s$, and $\alpha$ be the element of $\mathbb{Q}(\beta)$ defined by

$$
\alpha:=\sum_{n=1}^{+\infty} \frac{a_{n}}{\beta^{n}},
$$

where $\left(a_{n}\right)_{n \geqslant 1}$ is the eventually periodic sequence with preperiod $U$ and period $V$. Let $\xi$ be a real number, $0<\xi<1$, such that $d_{\beta}(\xi)=0 . b_{1} b_{2} \ldots b_{n} \ldots$, where $\left(b_{n}\right)_{n \geqslant 1}$ is such that there exists a positive integer $j>r+s$ satisfying:

(i) $a_{n}=b_{n}$, for $1 \leqslant n<j$;

(ii) $a_{j} \neq b_{j}$. 


\section{B. Adamczewski and J. Cassaigne}

Then,

$$
|\xi-\alpha|>\frac{1}{(s+1)^{l-1} \beta^{j+s+l-1}}
$$

Proof. The key observation is that for every non-negative integer $r$,

$$
\sum_{n \geqslant r+1} \frac{b_{n}}{\beta^{n}}<\frac{1}{\beta^{r}}
$$

Note that such an inequality is not, in general, satisfied by arbitrary representations in base $\beta$. However, it holds when one considers the $\beta$-expansion (as a by-product of the fact that the $\beta$-expansion arises from the greedy algorithm).

We first infer from the fact that the coefficients $b_{n}$ are non-negative and from (26) that

$$
\sum_{n=j-s+1}^{j} \frac{b_{n}}{\beta^{n}} \leqslant \sum_{n \geqslant j-s+1} \frac{b_{n}}{\beta^{n}}<\frac{1}{\beta^{j-s}}
$$

and, thus,

$$
b_{j-s+1} \beta^{s-1}+b_{j-s+2} \beta^{s-2}+\cdots+b_{j}<\beta^{s} .
$$

We also recall that by definition of the sequence $\left(a_{n}\right)_{n \geqslant 1}$, we have

$$
a_{n}=a_{n+t s}, \quad \text { if } n>r \text { and } t \geqslant 0 .
$$

We have now to distinguish two cases. We set $a_{j}=i$ and $b_{j}=m$, and we first assume that $i>m$. Then, $i$ is a positive integer and, the coefficients $a_{n}$ also being non-negative, we infer from (28) that

$$
\begin{aligned}
\alpha & =\sum_{n=1}^{+\infty} \frac{a_{n}}{\beta^{n}} \geqslant \sum_{n=1}^{j-1} \frac{a_{n}}{\beta^{n}}+\frac{a_{j}}{\beta^{j}}+\frac{a_{j+s}}{\beta^{j+s}}+\frac{a_{j+2 s}}{\beta^{j+2 s}} \\
& =\sum_{n=1}^{j-1} \frac{a_{n}}{\beta^{n}}+\frac{i}{\beta^{j}}+\frac{i}{\beta^{j+s}}+\frac{i}{\beta^{j+2 s}}>\sum_{n=1}^{j-1} \frac{a_{n}}{\beta^{n}}+\frac{i}{\beta^{j}}+\frac{i}{\beta^{j+s}},
\end{aligned}
$$

whereas thanks to condition (i) and inequality (26) we have

$$
\xi=\sum_{n=1}^{+\infty} \frac{b_{n}}{\beta^{n}}=\sum_{n=1}^{j-1} \frac{a_{n}}{\beta^{n}}+\frac{m}{\beta^{j}}+\sum_{n \geqslant j+1} \frac{b_{n}}{\beta^{n}}<\sum_{n=1}^{j-1} \frac{a_{n}}{\beta^{n}}+\frac{m+1}{\beta^{j}} \leqslant \sum_{n=1}^{j-1} \frac{a_{n}}{\beta^{n}}+\frac{i}{\beta^{j}} .
$$

This yields

$$
\alpha-\xi>\frac{i}{\beta^{j+s}} \geqslant \frac{1}{\beta^{j+s}}
$$

Now, let us assume that $m>i$. By condition (i), we have

$$
b_{j-s+1} b_{j-s+2} \ldots b_{j-1}=a_{j-s+1} a_{j-s+2} \ldots a_{j-1} .
$$

Moreover, since $a_{j}=i \leqslant m-1=b_{j}-1$, we deduce from (27) that

$$
P(\beta):=a_{j-s+1} \beta^{s-1}+a_{j-s+2} \beta^{s-2}+\cdots+a_{j}<\beta^{s}-1 .
$$


On the one hand, we infer from (28) that

$$
\begin{aligned}
\alpha & =\sum_{n=1}^{+\infty} \frac{a_{n}}{\beta^{n}}=\sum_{n=1}^{j} \frac{a_{n}}{\beta^{n}}+\sum_{n=1}^{+\infty} \frac{P(\beta)}{\beta^{j+n s}} \\
& =\sum_{n=1}^{j} \frac{a_{n}}{\beta^{n}}+\frac{P(\beta)}{\beta^{j+s}}+\sum_{n=2}^{+\infty} \frac{P(\beta)}{\beta^{j+n s}} \\
& =\sum_{n=1}^{j} \frac{a_{n}}{\beta^{n}}+\frac{P(\beta)}{\beta^{j+s}}+\frac{P(\beta)}{\beta^{j+s}\left(\beta^{s}-1\right)},
\end{aligned}
$$

and we thus derive from (29) that

$$
\alpha<\sum_{n=1}^{j} \frac{a_{n}}{\beta^{n}}+\frac{P(\beta)+1}{\beta^{j+s}} .
$$

On the other hand, we have

$$
\xi=\sum_{n=1}^{+\infty} \frac{b_{n}}{\beta^{n}} \geqslant \sum_{n=1}^{j} \frac{b_{n}}{\beta^{n}}=\sum_{n=1}^{j-1} \frac{a_{n}}{\beta^{n}}+\frac{m}{\beta^{j}} \geqslant \sum_{n=1}^{j-1} \frac{a_{n}}{\beta^{n}}+\frac{i+1}{\beta^{j}} .
$$

This gives

$$
\xi-\alpha>\frac{1}{\beta^{j}}-\frac{P(\beta)+1}{\beta^{j+s}}=\frac{Q(\beta)}{\beta^{j+s}},
$$

where $Q(\beta):=\beta^{s}-P(\beta)-1$. Moreover, $(29)$ implies that $Q(\beta)>0$. Note that $\beta$ is a Pisot or a Salem number of degree $l$ and that $Q$ is an integer polynomial of degree $s$ and of height at most $\lfloor\beta\rfloor$. Let us denote by $\beta=\beta_{1}, \beta_{2}, \ldots, \beta_{l}$ the algebraic conjugates of $\beta$. Then, $\beta$ being an algebraic integer, we have that $Q(\beta) \prod_{j=2}^{l}\left|Q\left(\beta_{j}\right)\right|$ is an integer. Since $Q(\beta)>0$, also $\left|Q\left(\beta_{j}\right)\right|>0$ for $2 \leqslant j \leqslant l$, and we obtain that $Q(\beta) \prod_{j=2}^{l}\left|Q\left(\beta_{j}\right)\right|>0$. Thus, $Q(\beta) \prod_{j=2}^{l}\left|Q\left(\beta_{j}\right)\right| \geqslant 1$. Moreover, for $j \geqslant 2$, $\left|Q\left(\beta_{j}\right)\right| \leqslant(s+1) \beta$, since $\left|\beta_{j}\right| \leqslant 1$. This yields

$$
Q(\beta) \geqslant((s+1) \beta)^{-l+1}
$$

and we thus infer from (30) that

$$
\xi-\alpha>\frac{1}{(s+1)^{l-1} \beta^{j+s+l-1}} .
$$

This ends the proof.

We now consider Theorem 7.2. The number field extension of Baker's result can be stated as follows. Let us assume that there exist $\eta>2$ and an infinite sequence $\left(\beta_{n}\right)_{n \geqslant 0}$ of distinct algebraic numbers lying in the same number field and such that: $\left|\xi-\beta_{n}\right| \ll H\left(\beta_{n}\right)^{-\eta}$ and

$$
\limsup _{n \rightarrow \infty} \frac{\log \left(H\left(\beta_{n+1}\right)\right)}{\log \left(H\left(\beta_{n}\right)\right)}<+\infty .
$$

Then, $\xi$ is not a $U$-number. We proceed as for the proof of Theorem 3.3 to construct a sequence $\left(\alpha_{n}\right)_{n \geqslant 0}$ such that $\left|\xi-\alpha_{n}\right| \ll H\left(\alpha_{n}\right)^{-2-\varepsilon}$, for a fixed positive $\varepsilon$. Then, we infer from Lemmas 5.1 and 8.2 that we can extract a suitable subsequence from $\left(\alpha_{n}\right)_{n \geqslant 0}$ to apply Baker's result.

We end this paper with some hints for the proof of Theorem 7.3. The basic idea consists of replacing the sequence of rationals $\left(p_{n} / q_{n}\right)_{n \geqslant 0}$ of the proof of Theorem 2.2 by a sequence $\left(\alpha_{n}\right)_{n \geqslant 0}$ of quadratic approximations, and then to use Lemma 8.1 with $t=2$.

Let $\xi:=\left[0, a_{1}, a_{2}, \ldots\right]$ be an automatic continued fraction and let us denote by $p_{n} / q_{n}$ the $n$th convergent to $\xi$. Thanks to Theorem 4.1, there exists a pair of morphisms $(\varphi, \sigma)$ and a letter $a$ such 


\section{B. Adamczewsiki and J. Cassaigne}

that $\mathbf{a}:=\left(a_{n}\right)_{n \geqslant 1}=\varphi\left(\sigma^{\infty}(a)\right)$. Since, by assumption, the letter $a$ occurs at least twice, there exists a finite word $U$ such that $a U a$ is a prefix of $\sigma^{\infty}(a)$. This implies that the sequence a begins for every integer $n$ with the word $U_{n} U_{n}^{\prime}$, where $U_{n}=\varphi\left(\sigma^{n}(a U)\right)$ and $U_{n}^{\prime}=\varphi\left(\sigma^{n}(a)\right)$. We set $\left|U_{n}\right|=s_{n}$ and $\left|U_{n}^{\prime}\right|=t_{n}$. Then, our quadratic approximants $\alpha_{n}$ are defined as the real numbers having a periodic continued fraction expansion with period $U_{n}$, that is, $\alpha_{n}:=\left[0, \overline{U_{n}}\right]$. We first observe that $\alpha_{n}$ is a root of the quadratic polynomial

$$
P_{n}(X):=q_{s_{n}-1} X^{2}+\left(q_{s_{n}}-p_{s_{n}-1}\right) X-p_{s_{n}}
$$

and, thus, $H\left(\alpha_{n}\right)<q_{s_{n}}$. On the other hand, we have

$$
\left|\xi-\alpha_{n}\right|<\frac{1}{q_{s_{n}+t_{n}}^{2}}
$$

since the first $s_{n}+t_{n}$ partial quotients of $\alpha$ and $\alpha_{n}$ are the same (see, for instance, [AB06a, Lemma $2]$ ). Moreover, the partial quotients of $\xi$ are bounded and we thus infer from classical inequalities for continuants that $q_{s_{n}+t_{n}}^{2} \gg q_{s_{n}}^{\eta}$ for a fixed $\eta>2$ (see, for instance, [AB06a, Lemmas 3 and 4]). We thus obtain

$$
|\xi-\alpha| \ll H\left(\alpha_{n}\right)^{-\eta} .
$$

Again, to finish the proof we only have to see that $\alpha_{n}$ is not a very good approximation of $\xi$ (this corresponds to the left-hand side of Lemma 8.1(ii)) and that there exists a subsequence of $\left(\alpha_{n}\right)_{n \geqslant 0}$ satisfying assumption (i) of Lemma 8.1. These conditions are ensured thanks to Lemma 5.1 and Lemma 8.3 below, the latter playing the role of Lemma 5.2 in the proof of Theorem 2.2.

Lemma 8.3. Let $M$ be a positive real number. Let $\alpha=\left[0, a_{1}, a_{2}, \ldots\right]$ and $\xi=\left[0, b_{1}, b_{2}, \ldots\right]$ be real numbers whose partial quotients are at most equal to $M$. Assume that there exists a positive integer $n$ such that $a_{i}=b_{i}$ for any $i=1, \ldots, n$ and $a_{n+1} \neq b_{n+1}$. Then, we have

$$
|\xi-\alpha| \geqslant \frac{1}{(M+2)^{3} q_{n}^{2}}
$$

where $q_{n}$ denotes the denominator of the $n$th convergent to $\alpha$.

This latter result corresponds to [AB06a, Lemma 5]. We thus refer the reader to that paper for a complete proof.

\section{ACKNOWLEDGEMENT}

The first author is grateful to Yann Bugeaud for useful discussions.

\section{REFERENCES}

Ada04 B. Adamczewski, Transcendance 'à la Liouville' de certains nombres réels, C. R. Acad. Sci. Paris Sér. I Math. 338 (2004), 511-514.

AB05 B. Adamczewski and Y. Bugeaud, On the complexity of algebraic numbers II. Continued fractions, Acta Math. 195 (2005), 1-20.

AB06a B. Adamczewski and Y. Bugeaud, On the Littlewood conjecture in simultaneous Diophantine approximation, J. London Math. Soc. (2) 73 (2006), 355-366.

AB B. Adamczewski and Y. Bugeaud, On the complexity of algebraic numbers I. Expansions in integer bases, Ann. of Math. (2), to appear.

AB06b B. Adamczewski and Y. Bugeaud, Dynamics for $\beta$-shifts and Diophantine approximation, Preprint (2006).

ABL04 B. Adamczewski, Y. Bugeaud and F. Luca, Sur la complexité des nombres algébriques, C. R. Acad. Sci. Paris Sér. I Math. 339 (2004), 11-14. 


\section{Diophantine PROPERTIES OF AUTOMATIC REAL NUMBERS}

AS92 J.-P. Allouche and J. O. Shallit, The ring of k-regular sequences, Theoret. Comput. Sci. 98 (1992), 163-197.

AS03 J.-P. Allouche and J. O. Shallit, Automatic sequences: theory, applications, generalizations (Cambridge University Press, Cambridge, 2003).

Bak64 A. Baker, On Mahler's classification of transcendental numbers, Acta Math. 111 (1964), 97-120.

BS76 L. E. Baum and M. M. Sweet, Continued fractions of algebraic power series in characteristic 2, Ann. of Math. (2) 103 (1976), 593-610.

BT98 R. M. Beals and D. S. Thakur, Computational classification of numbers and algebraic properties, Internat. Math. Res. Notices 15 (1998), 799-818.

Bug04 Y. Bugeaud, Approximation by algebraic numbers, Cambridge Tracts in Mathematics, vol. 160 (Cambridge University Press, Cambridge, 2004).

Cob68 A. Cobham, On the Hartmanis-Stearns problem for a class of tag machines, in IEEE Conference Record of the 1968 Ninth Annual Symposium on Switching and Automata Theory (IEEE Press, Piscataway, NJ, 1968), 51-60.

Cob72 A. Cobham, Uniform tag sequences, Math. Systems Theory 6 (1972), 164-192.

Dek77 F. M. Dekking, Transcendance du nombre de Thue-Morse, C. R. Acad. Sci. Paris Sér. I Math. 285 (1977), 157-160.

Eil74 S. Eilenberg, Automata, languages, and machines, vol. A (Academic Press, New York, 1974).

Gut68 R. Güting, Zur Berechnung der Mahlerschen Funktionen $w_{n}$, J. reine angew. Math. 232 (1968), $122-135$.

HS65 J. Hartmanis and R. E. Stearns, On the computational complexity of algorithms, Trans. Amer. Math. Soc. 117 (1965), 285-306.

Khi24 A. Khintchine, Einige Sätze über Kettenbrüche, mit Anwendungen auf die Theorie der Diophantischen Approximationen, Math. Ann. 92 (1924), 115-125.

Khi64 A. Khintchine, Continued fractions (University of Chicago Press, Chicago, IL, 1964).

KZ01 M. Kontsevich and D. Zagier, Periods, in Mathematics unlimited - 2001 and beyond (Springer, Berlin, 2001), 771-808.

Lev53 W. J. LeVeque, On Mahler's U-numbers, J. London Math. Soc. (2) 28 (1953), 220-229.

Lio44 J. Liouville, Sur des classes très étendues de quantités dont la valeur n'est ni algébrique, ni même réductible à des irrationnelles algébriques, C. R. Acad. Sci. Paris Sér. I Math. 18 (1844), 883-885 and 993-995.

Lot02 M. Lothaire, Algebraic combinatorics on words, Encyclopedia of Mathematics and its Applications, vol. 90 (Cambridge University Press, Cambridge, 2002).

LV82 J. H. Loxton and A. J. van der Poorten, Arithmetic properties of the solutions of a class of functional equations, J. reine angew. Math. 330 (1982), 159-172.

LV88 J. H. Loxton and A. J. van der Poorten, Arithmetic properties of automata: regular sequences, J. reine angew. Math. 392 (1988), 57-69.

Mah29 K. Mahler, Arithmetische Eigenschaften der Lösungen einer Klasse von Funktionalgleichungen, Math. Ann. 101 (1929), 342-366 (Corrigendum 103 (1930), 532).

Mah31 K. Mahler, Zur Approximation der Exponentialfunktion und des Logarithmus. Teil I, J. reine angew. Math. 166 (1931), 118-136.

Mah32a K. Mahler, Zur Approximation der Exponentialfunktion und des Logarithmus. Teil II, J. reine angew. Math. 166 (1932), 136-150.

Mah32b K. Mahler, Über das Mass der Menge aller S-Zahlen, Math. Ann. 106 (1932), 131-139.

Nis91 K. Niskioka, Algebraic independence measures of the values of Mahler's functions, J. reine angew. Math. 420 (1991), 203-214.

Ren57 A. Rényi, Representations for real numbers and their ergodic properties, Acta Math. Acad. Sci. Hungary 8 (1957), 477-493.

Rot55 K. F. Roth, Rational approximations to algebraic numbers, Mathematika 2 (1955), 1-20 (Corrigendum 2 (1955), 168). 
Sha99 J. O. Shallit, Number theory and formal languages, in Emerging applications of number theory, IMA Volumes (Springer, Berlin, 1999), 547-570.

Tur37 A. M. Turing, On computable numbers, with an application to the Entscheidungsproblem, Proc. London Math. Soc. (2) 42 (1937), 230-265 (Corrigendum 43 (1937), 544-546).

Wir60 E. Wirsing, Approximation mit algebraischen Zahlen beschränkten Grades, J. reine angew. Math. 206 (1960), 67-77.

Boris Adamczewski boris.adamczewski@math.univ-lyon1.fr

CNRS, Institut Camille Jordan, Université Claude Bernard Lyon 1, Bât. Braconnier, 21 avenue Claude Bernard, 69622 Villeurbanne cedex, France

Julien Cassaigne julien.cassaigne@iml.univ-mrs.fr

CNRS, Institut de Mathématiques de Luminy, Case 907, 163 avenue de Luminy, 13288 Marseille cedex 9, France 